\title{
Formación de personas para la transmisión de la fe: logros y desafíos de un currículum de teología pastoral
}

\author{
Fernando Berrios - Rodolfo Núñez \\ FACULTAD DE TEOLOGÍA \\ PONTIFICIA UNIVERSIDAD CATÓLICA DE CHILE \\ fberriosm@uc.cl - rnunezh@uc.cl
}

Los días 27 y 28 de agosto de 2014 se llevó a cabo en Santiago, en las dependencias de la Facultad de Teología de la Pontificia Universidad Católica de Chile, el $3^{\text {er }}$ Coloquio Internacional de Teología Pastoral. Se trata de una instancia de encuentro y reflexión que surgió en el marco de la creación y desarrollo de un currículum pastoral en esta facultad, y que se ha caracterizado desde sus orígenes por la búsqueda de un intercambio fecundo de experiencias y conocimientos entre académicos de diversas latitudes. El primer coloquio se llevó a cabo el año 2010, sobre el tema del estatuto epistemológico específico de la teología práctica en el conjunto de los saberes y disciplinas universitarias, y contó con la participación de representantes de los centros teológicos más importantes de América Latina: Pontificia Universi- dad Javeriana (Bogotá), Instituto Santo Ignacio-Facultad Jesuita de Teología (Belo Horizonte), Facultad Católica de Fortaleza-Brasil e Instituto Superior de Estudios Teológicos (Lima), además de profesores de nuestra Facultad. El segundo coloquio se realizó en 2011, sobre el tema del método de la teología pastoral en su cometido central de una lectura teológica de la realidad, y contó esta vez con aportes de especialistas de los ya mencionados centros teológicos latinoamericanos, como también del Instituto Católico de París.

En esta tercera ocasión quisimos vincular de un modo más específico la teología pastoral con el tema de la catequesis de iniciación cristiana como acción fundamental de la Iglesia para la transmisión de la fe. De singular relevancia ha sido en esta oportunidad la participación de un grupo de jóvenes 
académicos y doctorandos de diversas facultades teológicas de Alemania, bajo la guía del Prof. Dr. Albert Biesinger, ex profesor de la Universidad de Tübingen y gran especialista en el tema de la catequesis. En este marco de intercambio y de diálogo se inscriben las siguientes reflexiones a partir de una experiencia concreta de formación de personas para la transmisión de la fe, en el contexto de una carrera de pregrado de teología pastoral.

En una reflexión anterior sobre las experiencias iniciales en teología pastoral en la Pontificia Universidad Católica de Chile, los entonces directores del currículum de Estudios Pastorales utilizaban la imagen del ascenso de una montaña. Los tópicos principales eran el trabajo en equipo, el rol de cada uno de la cordada y, sobre todo, la existencia de un plan ideal previo que "luego debe someterse a la realidad del ascenso, integrando las sinuosidades del camino y el ritmo de las personas", para llegar a ser "el plan que, de hecho, se ejecuta en el ascenso (y) que se enriquece de la experiencia atravesada y de las peripecias enfrentadas. Para este último plan -concluía esta reflexión- es clave lo que significó la travesía. Eso permite el traspaso de experiencias para otros grupos que deseen emprender el ascenso" ${ }^{\prime}$. Precisamente en esta senda queremos ponernos en esta ocasión y específicamente en el ejercicio de hacer una necesaria pausa en el ascenso, para revisar el camino andado y preguntarnos en qué medida lo que hemos planeado para la continuación del camino es acertado.

Aquella primera reflexión tomaba, en efecto, en cuenta la experiencia fundante, pero poniendo acento en las opciones epistemológicas que había sido necesario tomar en ese momento. Es una reflexión, por tanto, que pone especial atención en la perspectiva teológica que constituye el punto de partida de este proyecto, para luego subrayar el carácter interdisciplinar de la teología pastoral y la dimensión comunicacional de su metodología. Pero al final los autores proponen algunas "propuestas para continuar el camino" ${ }^{2}$, en la línea específica de una "in-

Todas las citas tomadas de R. NúÑEZA. Toutin, " "Hoy ha llegado la salvación a esta casa» (Lc 19, 10). Aproximación dialogal e interdisciplinar a la complejidad del hoy: reflexiones acerca del núcleo de la teología práctica”, en Teología y Vida, 53/3 (2012) 373-395. 373.

2 R. Núñez-A. Toutin, " "Hoy ha llegado la salvación...”, 393-395. 
telección del reinado de Dios"3. Tres aspectos se plantean como fundamentales para ese propósito: 1) una confrontación del hoy con la praxis de Jesús que considere de un modo prioritario "las condiciones de posibilidad espirituales" del sujeto humano para sintonizar con esa praxis en su significación salvífica; 2) el rol de la Iglesia como "sacramento", es decir, en su carácter siempre referencial al único autor de la salvación que es Jesucristo; y 3) por último, la pregunta sobre la credibilidad de la Iglesia en cuanto a su capacidad de hacer una mediación del proyecto del Reino en Jesucristo para el mundo.

Recogiendo estos aspectos, quisiéramos hacer ahora un ejercicio distinto de balance y de bosquejo de grandes perspectivas para el desarrollo de un currículum de Teología Pastoral en nuestra facultad. Lo diverso de este nuevo balance radica en que su punto de partida no es ya de carácter epistemológico sino experiencial, tal como esta experiencia se refleja en el conjunto de las "evaluaciones tempranas" de los cursos que hemos venido haciendo en diálogo

3 R. NúNEzZ-A. Toutin, " "Hoy ha llegado la salvación...", 393, nota 28, citando a F. de Aquino Júnior, "O reinado de Deus como assunto da teología cristã", en REB 71 (2011) 54 . con los estudiantes en cada uno de los semestres.

Las "evaluaciones tempranas" se desarrollan como un diálogo entre los estudiantes de un mismo grupo-curso con todos los profesores de su nivel y la metodología se basa en una invitación a conversar, con toda trasparencia y en un clima de respeto, acerca de lo que los estudiantes y sus profesores consideran como fortalezas o debilidades en cada una de las asignaturas que se están desarrollando en el semestre. Por ser "tempranas", estas evaluaciones permiten a profesores y estudiantes corregir defectos o potenciar fortalezas en el tiempo restante del período académico. Se trata, pues, de un tipo de evaluación que no tiene como finalidad hacer un juicio sobre algo ya concluido, sino revisar, como decíamos al comienzo, los avances logrados o las dificultades experimentadas en la implementación del plan inicial de cada curso frente a "las sinuosidades del camino y el ritmo de las personas". El ejercicio de recoger aquí los resultados de estas evaluaciones nos permitirá, además, mostrar cuán importantes son ellas en esta facultad para la concreta organización académica de esta carrera.

En otro ámbito, queremos aprovechar también esta ocasión 
para revisar un aspecto fundamental de nuestro proyecto, a saber, las dificultades y los logros experimentados en el complejo camino del diálogo interdisciplinar como dimensión esencial de la teología pastoral, y de un modo especial en los "talleres" de síntesis que se realizan en cada semestre. En general, es nuestra intención que estas reflexiones puedan ser una oportunidad para compartir una experiencia académica relevante y contribuir así a otros centros teológicos interesados en el desarrollo de esta disciplina teológica particular.

1. LOGROS Y DESAFÍOS PENDIENTES: LA VOZ DE NUESTROS ESTUDIANTES

¿Qué nos han dicho en los últimos años las "evaluaciones tempranas" acerca de lo que nuestros estudiantes valoran como logros o dificultades de la reflexión teológicopastoral en nuestra carrera y más específicamente en el aula?

\section{Logros}

Partamos por los logros, considerándolos como indicadores de aspectos que habría que cultivar y profundizar en nuestro quehacer teológico.

1. Espacios de reflexión y diálogo. En diversas asignaturas se valora y se agradece especialmente cuando los docentes logran articular el trabajo de aula y el estudio personal, más que como mera transmisión de contenidos e informaciones, como un espacio para la confrontación de ideas, la crítica y la autocrítica sobre la Iglesia, la teología y las prácticas pastorales, y para el planteamiento de buenas preguntas sobre estas y otras temáticas relevantes.

2. Metodologias activas y participativas. Un enfoque crítico y autocrítico del quehacer teológico y de las prácticas pastorales se traduce necesariamente en metodologías de enseñanzaaprendizaje basadas en la construcción de un pensamiento autónomo, aunque desde el diálogo y la confrontación con otros. El trabajo de aula basado en este principio, favorece en el desarrollo de un pensamiento personal que no eluda la responsabilidad de una fundamentación sólida, en diálogo con perspectivas diversas o complementarias a las propias.

\section{Bases sólidas para la articulación} de teoría y práctica. Se busca con ahínco evitar una dicotomía entre estos dos ámbitos. La práctica pastoral no se concibe como un espacio de mera "aplicación” de ideas concebidas a 
priori y de modo abstracto. El pensamiento teológico-pastoral se concibe, por el contrario, como algo que se construye desde la realidad misma, con ella y desde ella, haciendo el esfuerzo de articular categorías teológicas en diálogo con categorías de otras disciplinas.

4. Esfuerzo de sintesis a partir de los contenidos abordados en las diversas asignaturas. Se valora que con esto se busque evitar que los saberes particulares sean considerados como apartamentos estancos y que se busque más bien identificar sus puntos de contacto, en función de una mirada integradora sobre el ser humano, el cristianismo y la Iglesia en el mundo. Este esfuerzo de síntesis los alumnos suelen destacarlo en alguno de los Talleres, pero también en varias de las asignaturas. En varias ocasiones los estudiantes utilizan la expresión "eje articulador", como elemento que integra entre sí diversas aproximaciones dentro de un mismo semestre; por ejemplo, el eje "antropología relacional" o el eje "pueblo de Dios" en el tercer semestre. Idealmente, este esfuerzo de síntesis debería reflejarse también, como un logro importante del estudiante, en el examen final de grado. Este as- pecto, que los estudiantes destacan en diversas asignaturas, está también relacionado con un esfuerzo metodológico tendiente a superar la dispersión y a establecer, por el contrario, nexos internos que permitan pensar con coherencia. Un desafío importante vinculado a este aspecto es que la ayuda que den los profesores para este fin, debe hacerse de tal manera que los estudiantes puedan hacer $s u$ propia sistematización y no se limiten, por tanto, a reproducir la que aquellos reflejan en su labor docente.

\section{Pastoralidad del quehacer teoló-} gico. Este aspecto es el que da su impronta a esta carrera. No siempre, pero en ciertos momentos se logra esta pastoralidad, sobre todo cuando en el aula se dan orientaciones que ayudan a los estudiantes a hacer síntesis y sistematizaciones que vinculan los contenidos de las asignaturas con su propia práctica pastoral. Por ese camino se alcanza un doble propósito fundamental de esta carrera: por una parte, avanzar hacia una práctica pastoral basada en una reflexión teológica sólida; y por otra, aprender a reflexionar teológicamente desde y para la existencia cristiana y la práctica pastoral. Cuando los estu- 
diantes experimentan que el estudio no es un distractor sino un aliado de la acción pastoral, entonces esta carrera comienza a mostrar su sentido.

6. La importancia del fundamento eclesiológico de la teología pastoral. Más exactamente, el conocimiento a fondo de la eclesiología del Concilio Vaticano II, sobre todo en la clave del pueblo de Dios, permite comprender más cabalmente el desafío de la teología pastoral como una reflexión compleja que trasciende el terreno de las simples estrategias y que solo es posible como tarea abierta para el conjunto de la Iglesia, con su diversidad de carismas y ministerios, en su inserción en el mundo. La teología pastoral supone así, como una auténtica condición de posibilidad, la superación de toda concepción jerarcológica de la Iglesia.

7. La conciencia creciente acerca de la necesidad y también de la complejidad del diálogo interdisciplinar. En esta carrera los estudiantes han podido experimentar esta doble convicción: la teología pastoral solo puede llevarse a cabo desde la interdisciplinariedad; pero no es menos cierto que este es un desafío mayor y difícilmente realizable.
La interdisciplinariedad, si se la quiere practicar con seriedad y rigor, no es fácil, pues exige progresar en el conocimiento de la propia disciplina -en este caso, la teología- a la par que en el conocimiento de las lógicas y las categorías básicas de otras ciencias.

8. Dominio y solidez de los conocimientos disciplinares de los docentes. En varias ocasiones, en las evaluaciones tempranas los estudiantes destacan especialmente el amplio y profundo conocimiento de sus profesores en sus respectivos campos. A veces la valoración de las capacidades didácticas de los profesores por parte de los estudiantes, no va a la par. Con todo $\mathrm{y}$ aunque esto indica también una debilidad, es destacable la importancia que tiene para nuestros estudiantes la preparación científica de sus docentes.

9. La importancia de la didáctica en el aula y en los procesos de aprendizaje individuales. Este punto es la contracara del anterior. Se destaca especialmente cuando el docente, además de conocimientos amplios y sólidos y de una actitud de apertura y diálogo (que se subraya como lo más importante para el proceso de enseñanza-aprendizaje) 
logra también una adecuada organización de los contenidos del curso y sabe recurrir a adecuadas y diversas estrategias para el intercambio de conocimientos y reflexiones. Parte importante de estas estrategias son aquellas que ponen a nuestros estudiantes en contacto directo con expresiones de la cultura, tales como la prensa escrita, la literatura, el teatro o el cine.

\section{Desafíos pendientes}

Nuestros estudiantes han destacado también los siguientes aspectos entre las principales limitaciones por superar. Los tres primeros son de índole metodológica.

1. Exceso de información en las clases. Este aspecto denota una falta de conciencia acerca de las características culturales de la "sociedad de la información" en la que vivimos en la actualidad. La mayoría de los profesores fuimos formados en nuestras respectivas disciplinas en un tiempo previo al explosivo desarrollo de las tecnologías de la información. Por ello, a veces todavía nos comportamos como si parte importante de nuestro rol sea la transmisión material de la información, obviando el hecho de que nuestros estudiantes tienen múltiples formas de acceder a ella. Es necesario que los docentes, en general, avancemos en un rol de orientadores para la obtención y el procesamiento personal de esa información, y que en consecuencia privilegiemos en el aula un trabajo de análisis y de reflexión a partir de la misma, más que de su transmisión.

2. Criterios y formas de evaluación inadecuados. Este aspecto es, probablemente, una consecuencia de lo anterior. Es necesario privilegiar la evaluación de procesos cognitivos (tales como la reflexión, la capacidad de síntesis, la relación) más que del acopio de información. Nuestras estrategias de evaluación deben ser más coherentes con los objetivos del currículum, que da especial importancia a los diagnósticos y a la propuesta de acciones pastorales apropiadas al medio diagnosticado.

3. Insuficiente participación en la evaluación de los trabajos finales de investigación, por parte de los responsables de los ambientes pastorales (parroquias, colegios, movimientos, etc.) en que esos trabajos son elaborados. Dado que uno de nuestros requisitos para la obtención del grado académico es una investigación con implicancias propiamente 
pastorales, debería adjuntarse al trabajo final un informe elaborado por el responsable de la pastoral en que este fue llevado a cabo. El informe debería dar indicaciones acerca de la pertinencia del tema y de la metodología del trabajo para la acción pastoral en función de la cual fue realizada.

Finalmente, nuestros estudiantes han señalado algunos temas de fondo que habría que profundizar o corregir en un currículum de esta índole:

4. Herramientas todavía insuficientes para incentivar, sostener y evaluar la calidad del trabajo en equipo. Este aspecto se capta tanto en ciertas actividades grupales de las asignaturas, como de un modo especial en los trabajos de los Talleres. La teología pastoral se ordena en gran medida a insertar a las personas en dinámicas comunitarias de crecimiento y desarrollo; pero no debe obviarse que este esfuerzo lo hacemos en un contexto cultural que por todas las vías promueve más bien la búsqueda de éxitos individuales. Por lo mismo, no han sido escasas en nuestra carrera experiencias poco satisfactorias de trabajo en equipo, y ello debe llamar a una reflexión que toca a un aspecto esencial de nuestro proyecto.

5. Déficit filosófico y de conocimiento de otras ciencias humanas. Dado que es posible que alumnos ingresen a la carrera sin una formación filosófica básica, esto se convierte en un déficit muy difícil de superar y que afecta también al quehacer del docente y de los estudiantes que sí tienen esa formación. Influye sobre todo en el nivel de profundidad que se pueda alcanzar en muchos temas de importancia, y sobre todo en las posibilidades reales de un diálogo interdisciplinar.

6. Todavía escasa contextualización latinomericana de los contenidos. En rigor, este es un problema que no se da solo en nuestro currículum, sino en general en las carreras de teología en Latinoamérica. Dado el criterio ampliamente extendido de que en el pregrado es necesario privilegiar una visión de conjunto sobre los diversos tratados teológicos, muchas veces por razones de escasez de tiempo la toma de contacto con las perspectivas específicas de la reflexión teológica y del Magisterio latinoamericanos es escasa o superficial. Consideramos que este no es un tema cerra- 
do y que es necesario y prioritario dar con buenas fórmulas para una mejor integración de lo latinoamericano en nuestro currículum.

Otro gran ámbito en que es importante hacer un balance es en el terreno del trabajo interdisciplinar, sobre todo en los Talleres semestrales. A continuación nos referiremos brevemente a este tema.

2. EL ARDUO CAMINO DE LA INTERDISCIPLINARIEDAD EN UN CURRÍCULUM PASTORAL

En el proceso de pensar la elaboración y la gestión de nuestra carrera de Licenciatura en Estudios Pastorales, ha ido surgiendo de un modo progresivo la necesaria diferenciación entre el pensar teológico y el fenómeno religioso. Ya no se trataba -al menos, no exclusivamente- de aproximarse a estudiar lo religioso como una dimensión de la conducta humana, sino de hacerse cargo de la comprensión de lo religioso por parte de una disciplina cuyos supuestos ontológicos y epistemológicos generan un paisaje de posibilidades distinto a las ciencias humanas. La teología católica desde su núcleo duro razona desde el dato-experiencia de la fe en Dios manifestado en Jesucristo, por su Espíritu, para la salvación de la humanidad. Pero elabora sus afirmaciones desde soportes deudores del pensamiento filosófico, produciéndose así una alta exigencia al momento de construir y establecer los puentes con otras disciplinas.

El discurso de las ciencias humanas, cuyo horizonte apunta a la comprensión del quehacer humano desde su condición intrasíquica, pasando por sus dinámicas interaccionales y alcanzando sus producciones globales en el plano de la cultura, habitualmente aborda lo religioso como una manifestación -entre otras- de la condición humana y su existencia. Eso incluye al núcleo del pensamiento teológico, que es asumido como una manifestación cultural. Esto se entiende en el contexto de una concepción, ya consagrada, de la autonomía de los saberes científicos con respecto a la cosmovisión o la antropología que pueda estar vinculada a la fe religiosa en cuanto tal.

Para poner un ejemplo, este encuentro de modelos de realidad que se da entre la teología y las ciencias humanas ha tenido momentos muy difíciles con los modelos psicoanalíticos, que han terminado patologizando la comprensión religiosa, o con la sociología marxista, para la cual lo religioso termina siendo un instrumento más para la opresión ejerci- 
da por las clases dominantes. Solo en los últimos cuatro decenios en la psicología se vislumbran líneas de encuentro, en particular con la denominada psicología transpersonal ${ }^{4}$. En este campo del conocimiento la dimensión espiritual aparece como una posibilidad de desarrollo humano desde lo comprendido como los fenómenos de expansión de conciencia. Proposiciones comprensivas del tipo "el gran nido del ser" ${ }^{5}$, marcan un punto de inflexión en los presupuestos ontológicos y epistémicos con los cuales se ha desenvuelto el pensar humanista en Occidente.

De otra parte, en las ciencias humanas se registra la irrupción del paradigma constructivista que propone nuevas aproximaciones a temas como visión del mundo, concepción del conocimiento, cri-

4 Esta aproximación de la psicología es de fines de la década del 60 y se aboca al estudio de los denominados "fenómenos de expansión de conciencia", en donde tienen cabida las experiencias referidas situaciones gestacionales y de parto y a contactos con mundos espirituales. Cf. S. Grof, El Poder curativo de las crisis (Ed. Kairós, Barcelona, 1993); S. Grof, Psicología Transpersonal: nacimiento y muerte en psicoterapia (Ed. Kairós, Barcelona, 2001); S. Krippner, La experiencia mistica (Ed. Kairós, Barcelona, 1992); K. WILBER, Breve historia de todas las cosas (Ed. Kairós, Barcelona, 2000).

5 Cf. K. Wilber, Breve historia... terios para validar el conocimiento y visión del ser humano ${ }^{6}$. Esto crea una gran posibilidad de diálogo con el pensamiento teológico, no porque valide particularmente sus proposiciones, sino más bien porque no las invalida a priori y en esa medida es capaz de establecer opciones de diálogos cooperativos, cuyo gran caudal de posibilidades está por venir.

\section{Perspectivas}

Nuestra impresión es que es posible establecer estos vínculos, en la medida en que se tome conciencia de los límites y puntos de contacto entre las disciplinas. Es posible formular opciones de trabajos conjuntos, pero para lograrlo se deben escoger los enfoques de las ciencias humanas con los cuales este diálogo resulta posible. Esto implica que ambos dialogantes deben ser permeados por las propuestas que aportan a una aproximación a la realidad humana, tanto en sus coordenadas psíquicas como socioculturales y espirituales. Se debe buscar un dinamismo conversacional en donde las disciplinas no se estén permanentemente demandando "cartas de ciudadanía", para

6 Para mayores antecedentes sobre este punto, cf. L. Botella y G. Feixas, Teoría de los constructos personales: Aplicaciones a la práctica psicológica (Editorial Laertes, Barcelona, 1998). 
referirse al objeto de estudio, sino que se reconozcan mutuamente en su validez e intercambien sus caudales interpretativos. Resultaría del todo conveniente trabajar en la elaboración de categorías conceptuales comunes, que permitan la producción de conocimientos útiles para ambas líneas disciplinarias, como resulta ser, por ejemplo, la categoría persona en el encuentro entre la filosofía y la teología.

La elaboración de este tipo de categorías compartidas permitiría la reflexión del evangelio desde una realidad psicosocio histórico y cultural y la comunicación del evangelio al hombre y sus circunstancias, pues desde este lugar se posibilita el encuentro en donde la relación del hombre con Dios es comprendida desde los diversos planos analíticos aportados por las ciencias humanas y estas desarrollan sus hipótesis interpretativas considerando activamente la experiencia de la fe. Ahora bien, supuesta la posibilidad de creación de categorías comprensivas comunes, esto se puede llevar adelante en una forma de encuentro colaborativo, evitando que se convierta en una suerte de reduccionismo en donde una disciplina termine explicándose por la otra, o una simple "traducción" de categorías que solo implicaría un intercambio de "diccionarios". La idea es más bien apuntar a una forma de reinterpretación, asumiendo como posibilidad razonable el contenido de las aseveraciones formuladas por ambas dimensiones del saber.

De este modo podremos superar también un antagonismo estéril, en que los peligros tienen que ver con las posibles descalificaciones mutuas o el reduccionismo de un modelo en el otro. Hablamos de alcanzar una forma de trabajo en donde la relación del hombre con Dios se lleve adelante enfatizando el conocimiento de las condiciones de posibilidad en el ser humano para escuchar y acoger una posible comunicación de Dios para él.

Nuestra experiencia de interdisciplinariedad en los Talleres

Los denominados Talleres de nuestro currículum pastoral aparecen como una expresión empírica de este encuentro conceptual. En ellos se refleja un esfuerzo por crear dichos diálogos y avanzar en estas fórmulas desde una lógica inductiva, aprendiendo de los buenos resultados y de lo que ha ido apareciendo como menos logrado.

Los Talleres son dirigidos por dos profesores, uno de la especialidad de las ciencias humanas que interese abordar y otro de la especialidad de la teología con la cual resulta de mayor pertinen- 
cia articular las conversaciones. Los equilibrios en el diálogo entre disciplinas se producen en dos momentos: inicialmente, en la planificación del programa y en la planificación de cada una de las clases. Allí se dan las conversaciones que construyen el encuentro entre modalidades comprensivas y performativas de la realidad, como resultan ser las ciencias humanas y la ciencia teológica en su expresión de teología pastoral. Un segundo momento se presenta durante la ejecución de las clases mismas, en donde la exposición de uno u otro docente es constantemente complementada con la mirada que surge desde la otra disciplina. Esto exige un ambiente de confianza mutua entre los profesionales a cargo y una competencia suficiente de cada uno en el campo del otro, para poder hacer de contraparte informada y articular así, en tiempo real, el diálogo entre dos formas de conocimiento.

En el primer Taller, denominado Estudio y aprendizaje significativo en teología, trabajan en conjunto un docente de la psicología educacional y un teólogo. Su propósito general es avanzar en la construcción de buenas opciones para enseñar el Evangelio, haciendo uso de las proposiciones de la psicología y permitir así el aprendizaje significativo en las personas.
De este modo se busca llegar a propuestas concretas para una mejor comunicación del Evangelio a las personas hoy.

El segundo Taller, que tiene como tema la Dimensión personal de la acción pastoral, pretende llevar a los participantes a la exploración del origen de los comportamientos de fe, profundizando en los factores que intervienen en su desarrollo y en su madurez y construyendo categorías para mejor intervenir en dichas conductas. La lectura teológica se hace desde Teología fundamental y la Antropología cristiana.

En el tercer Taller, sobre la $D i$ mensión comunitaria de la acción pastoral, se incorpora el trabajo con las categorías de la realidad social en la comprensión de la fe. La creencia es vista como un fenómeno social, con las dinámicas propias que esto implica y es complementada con las comprensiones teológicas que surgen de la $\mathrm{fe}$, es decir, con una dimensión comunitaria que le es propia. Lo social y lo eclesial se iluminan recíprocamente para llevar así adelante este ejercicio de encuentro entre la sociología y la teología.

En el cuarto taller, que aborda la Dimensión comunicacional de la acción pastoral, los estudiantes deben aprender la lógica opera- 
cional de los medios de comunicación masivos. Para ello, deben ejercitarse muy concretamente en la elaboración de trabajos en la técnica de algunos de estos medios (radio, televisión, internet $\mathrm{u}$ otros). El propósito de superar un entrenamiento meramente técnico se logra mediante un enfoque teológico trinitario.

En el quinto taller, la Dimensión cultural de la acción pastoral, en la forma específica de una antropología cultural, entra en diálogo con la teología fundamental y con el Magisterio, preparando así al estudiante para reconocer las variables que cada realidad humana incorpora en la interpretación del Evangelio. Además, aquí se inicia el proyecto de investigación sobre una realidad pastoral que los estudiantes deberán llevar adelante en el semestre siguiente.

Finalmente, en el sexto taller de Investigación pastoral los futuros teólogos deben llevar a cabo una investigación sobre un tópico pastoral relevante para su formación profesional. Luego de escoger el tema y utilizando una metodología cualitativa, deben explorar la realidad para contrastarla con categorías del pensar teológico. De ese modo, deben convertir el conocimiento producido en una he- rramienta útil para intervenciones teológico-pastorales.

Principales logros de la metodología interdisciplinar de los Talleres

1. En general ha ayudado a los estudiantes aprender a mirar la realidad en la que realizarán su trabajo teológico pastoral con categorías disciplinarias complementarias a las teológicas, que apuntan a un fin semejante, a saber, comprender lo humano para intervenir procurando su máximo desarrollo.

2. Ha entregado a los estudiantes criterios que le permitirán justificar la realización (o la inhibición) de determinadas prácticas en el trabajo con personas y con comunidades. Esto ha sido posible gracias a que las ciencias humanas pueden aportar indicadores de eficiencia y eficacia de las tareas que se emprenden.

3. Ha aportado un lenguaje novedoso y habilitante para el teólogo pastoral, que le permitirá intercambiar comprensiones y criterios de acción con otros profesionales del ámbito del trabajo con personas, comunidades u organizaciones.

4. Ha contribuido también a aprender a mirar la realidad desde otras perspectivas analíticas. Esto, ciertamente, facilita el des- 
centramiento y la flexibilidad de las comprensiones propias de la mirada eclesial y teológica y otorga así una mayor profundidad y potencial de adaptación en función de los objetivos propios de la teología pastoral.

Dificultades y desafíos pendientes

1. Al importante esfuerzo intelectual que implica para el estudiante de teología el aprendizaje de una epistemología distinta, se debe agregar la no menor tensión conceptual que conlleva el diálogo con supuestos ontológicos y antropológicos que contradicen o cuestionan directamente aquellos que son propios del modelo cristiano-católico.

2. Además de asumir nuevos horizontes analíticos, se debe sumar el aprendizaje de "nuevos diccionarios", propios de cada disciplina. Esto tiene una condición ambivalente, pues por una parte enriquece la perspectiva del estudiante, pero al mismo tiempo lo somete, en un lapso más bien breve, al aprendizaje de "lenguas novedosas", con la alta exigencia que esta tarea demanda.

3. Los docentes a cargo de los talleres deben mostrar un alto grado de sintonía, tanto entre ellos -en cuanto representantes de disciplinas diversas- como con los alumnos. Cuando esto no se logra, la calidad del taller se resiente significativamente. El esfuerzo de diálogo interdisciplinar no se puede falsear y se encuentra siempre expuesto a una comprobación inmediata en el aula.

4. El ideal del Taller, que apunta a alcanzar al menos una cierta síntesis de los contenidos centrales de los diversos cursos del semestre en que se dicta, no se ha podido lograr hasta ahora de una forma óptima, debido, sobre todo, a que no se cuenta aún con las redes conceptuales y las didácticas que permitan una integración bien lograda al respecto.

5. No se ha logrado tampoco, hasta ahora, una suficiente estabilidad del cuerpo docente a cargo de los Talleres. Esta estabilidad mínima aparece como una condición de posibilidad para ir construyendo soluciones didácticas verdaderamente adecuadas a las exigencias del trabajo interdisciplinar en que estos talleres están basados.

A MODO DE CONCLUSIÓN: LECCIONES PARA SEGUIR EN CAMINO

De todo lo anterior queda claro que nuestro currículum pastoral se 
ha desplegado como un esfuerzo formativo con un doble propósito: por una parte, avanzar hacia una práctica pastoral basada en una reflexión teológica sólida; y por otra, ayudar a aprender a reflexionar teológicamente desde y para la existencia cristiana y la práctica pastoral. Ello nos exige seguir avanzando en el desarrollo de un pensar teológico que articule vínculos colaborativos de calidad con las ciencias humanas y cultive adecuadas estrategias didácticas.

Para avanzar de un modo certero en esa dirección es necesario mantenerse activos en la potenciación de las duplas pedagógicas a cargo de los talleres. La experiencia nos ha mostrado que del éxito de este ejercicio pedagógico depende la posibilidad de desarrollar modelos de comprensión y de articulación de la interdisciplina en función de las prácticas de evangelización.

Junto a lo anterior, en el plano estrictamente teológico no hay que claudicar en el esfuerzo por hacer aplicable la reflexión a los contextos, en todo el proceso for- mativo. Esto quiere decir que cada uno de los cursos debería plantearse, por parte del docente y de los estudiantes, en la perspectiva que los contenidos fundamentales puedan tener una relevancia directa o indirecta para el quehacer pastoral y que, en consecuencia, pueda ser efectivamente posible su comunicación en ambientes no académicos.

De un modo especial, aparece como un gran desafío pendiente el incorporar con mayor claridad y con mayor certeza metodológica las perspectivas de la teología que se articula desde el contexto latinoamericano. Un progreso en esta línea favorecerá la identificación de nuestros estudiantes con su realidad próxima y facilitará así la vinculación de los contenidos de los estudios con la realidad sociocultural en que se despliega o se desplegará la acción pastoral. Un complemento importante de este aspecto será una más clara y vigorosa vinculación del proceso formativo de nuestro currículum con los ambientes pastorales que frecuentan nuestros estudiantes. 
